\title{
An Efficient One-Pot Three-Component Synthesis of Novel Sulfanyl Tetrazoles Using Ionic Liquids
}

\author{
Sankari Kanakaraju, Bethanamudi Prasanna, and G. V. P. Chandramouli \\ Department of Chemistry, National Institute of Technology, Warangal 506 004, India \\ Correspondence should be addressed to G. V. P. Chandramouli; gvpc2000@gmail.com
}

Received 13 June 2012; Accepted 11 August 2012

Academic Editor: Mohamed Afzal Pasha

Copyright (C) 2013 Sankari Kanakaraju et al. This is an open access article distributed under the Creative Commons Attribution License, which permits unrestricted use, distribution, and reproduction in any medium, provided the original work is properly cited.

An efficient, simple, and environmentally benign method for the synthesis of novel sulfanyl tetrazoles has been achieved by onepot three-component reaction of phenacyl bromides/3-(2-bromoacetyl)coumarins with $\mathrm{KSCN}$ and $\mathrm{NaN}_{3}$ using $\left[\mathrm{Bmim} \mathrm{BF}_{4}\right.$ ionic liquid. It could be reused and recycled for four runs without significant loss of product yield.

\section{Introduction}

Over the years, multicomponent reactions (MCRs) have become increasingly popular tools to ensure sufficient molecular diversity and complexity. They have gained significant popularity in recent years due to their atom-economy and straightforward reaction design due to substantial minimization of waste, labour, time, and cost $[1,2]$.

Tetrazoles play an important role in coordination chemistry as ligands, in medicinal chemistry as stable surrogates for carboxylic acids and in materials applications, including explosives, rocket propellants, and agriculture [313]. There is a particular interest for the synthesis of 5alkyl/arylthiotetrazoles as these thiotetrazoles are having powerful activating property than the corresponding 5akyl/aryltetrazoles used for synthesis of DNA and RNA [14, 15]. The presence of alkylthio group makes the tetrazole ring more acidic than the corresponding 5-alkyltetrazoles which improves its ability to act asan activator [16].

On the other hand, coumarins are heterocyclic organic compounds which constitute an important group of natural products having varied biological activities such as antitumor, antiinflammatory, antiviral, CNS, antioxidant, and anti-HIV activities [17-20].

Thus, in view of the diverse activity of coumarins and tetrazoles, as a part of our continuing research work on the synthesis of novel heterocyclic compounds, we thought to synthesize the compounds containing coumarin-substituted sulfanyl tetrazoles.

The most convenient method of synthesizing tetrazoles is the addition of azide ions to nitriles. Earlier reported methods for the synthesis of 5-substituted tetrazoles suffer from drawbacks such as the use of strong Lewis acids, or expensive and toxic metals, and the in situ generated hydrazoic acid which is highly toxic and explosive [21-24].

Several syntheses of 5-substituted tetrazoles have been reported through the $[2+3]$ cycloaddition of nitriles using $\mathrm{NaN}_{3}$ or TMSN ${ }_{3}$ in the presence of catalysts such as $\mathrm{ZnCl}_{2}$ [25], $\mathrm{AlCl}_{3}$ [26], $\mathrm{BF}_{3}-\mathrm{OEt}_{2}$ [27], $\mathrm{Pd}\left(\mathrm{PPh}_{3}\right)_{4}$ [28], $\mathrm{FeCl}_{3}-\mathrm{SiO}_{2}$ [29], TBAF [30], Zn/Al hydrotalcite [31], $\mathrm{ZnO}$ [32], and $\mathrm{Cu}_{2} \mathrm{O}$ [33]. Recently, LeBlanc and Jursic [34] and Demko and Sharpless [35] reported the synthesis of sulfanyl tetrazoles. Although these methods were quite useful, but having some limitations such as use of toxic solvents and use of homogeneous Lewis acid catalyst like $\mathrm{ZnBr}_{2}$ which could not be recycled from the reaction mixture. All the above methods require prolonged reaction times. Therefore, we thought of developing more efficient and convenient method which is free from all the above drawbacks.

\section{Results and Discussion}

In recent times, ionic liquids have attracted increasing interest in the context of green synthesis. These ILs have shown 
great promise as an alternative to conventional solvents due to their unique properties of nonvolatility, nonflammability, thermal stability, recyclability, and controlled miscibility [36-40]. Butylimidazolium salts ILs have already been demonstrated as efficient catalysts and solvents for various organic transformations [41-44].

Our literature survey revealed that till now there were no methods reported in the literature for the synthesis of sulfanyl tetrazoles via one-pot three-component reaction using ionic liquids. As part of our ongoing research work on the developments of new routes to heterocyclic system in ionic liquids $[45,46]$, we, herein, wish to report a simple and efficient procedure for one-pot threecomponent synthesis of novel sulfanyl tetrazoles by using 3-(2-bromoacetyl)coumarins/phenacyl bromides, KSCN and $\mathrm{NaN}_{3}$ in the presence of $[\mathrm{Bmim}] \mathrm{BF}_{4}$ ionic liquid at $100^{\circ} \mathrm{C}$ to afford title compounds in good yields. (Schemes 1 and 2).

Ionic liquids (ILs) based on butylimidazolium salts were tested as solvents. In order to optimize the reaction conditions, a model reaction was performed using 4chlorophenacyl bromide 5 b, KSCN and $\mathrm{NaN}_{3}$ using various ionic liquids.

A mixture of 4-chlorophenacyl bromide $5 \mathbf{b}$ and $\mathrm{KSCN}$ in ionic liquid was stirred at RT for 10 minutes. After completion of the reaction, $\mathrm{NaN}_{3}$ was added and the reaction was continued for $12 \mathrm{~h}$, there was no product formation as observed by TLC, whereas at $60^{\circ} \mathrm{C}$ the reaction proceeded but not to completion even after $8 \mathrm{~h}$. Consequently, the reaction temperature was optimized at $100^{\circ} \mathrm{C}$, which gave the sulfanyltetrazole as the sole product. The results were summarized in Table 1. As can be seen from Table 1, the best result was obtained when the reaction was carried out in $[\mathrm{Bmim}] \mathrm{BF}_{4}$ at $100^{\circ} \mathrm{C}$ (Table 1 , entry 4 ). The reaction using $[\mathrm{Bmim}] \mathrm{BF}_{4}$ proceeded in higher yield and shorter reaction time than that using another ionic liquids as reaction media.

The recyclability of the ionic liquid was also investigated using the above model reaction. After completion of the reaction, the mixture was poured into water and stirred thoroughly. The solid product was isolated by filtration, and the filtrate containing ionic liquid was extracted with ethyl acetate $(2 \times 20 \mathrm{~mL})$ to remove nonionic organic impurities. Then, the water was evaporated under reduced pressure and the recovered ionic liquid was dried at $80^{\circ} \mathrm{C}$ under vacuum for $2 \mathrm{~h}$ and reused in the next reaction. The procedure was repeated, and the results indicated that the ionic liquid could be reused for four times without evident loss in the yield of the product (Table 2). The scope and the generality of the present method were further demonstrated by the reaction of various phenacylbromides/3-(2-bromoacetyl)coumarins with KSCN and $\mathrm{NaN}_{3}$. In all cases, up to quantitative yields in reasonable reaction times were obtained (Table 3 ). All the synthesized compounds were characterized by m.p, elemental analysis, IR, ${ }^{1} \mathrm{H}$ NMR, mass, and ${ }^{13} \mathrm{C}$ NMR data.

\section{Conclusion}

In summary, we have developed an efficient, practically convenient, and ecologically safe method for the synthesis
TABLE 1: Synthesis of $\mathbf{6 b}$ in various ILs at $100^{\circ} \mathrm{C}$.

\begin{tabular}{lccc}
\hline Entry & Solvent & Time $(\mathrm{h})^{\mathrm{a}}$ & Yield $(\%)^{\mathrm{b}}$ \\
\hline 1 & {$[\mathrm{Bmim}] \mathrm{Cl}$} & 7 & 74 \\
2 & {$[\mathrm{Bmim}] \mathrm{Br}$} & 6.5 & 66 \\
3 & {$\left[\mathrm{Bmim}^{\mathrm{a}} \mathrm{PF}_{6}\right.$} & 8 & 48 \\
4 & {$\left[\mathrm{Bmim}_{\mathrm{Bm}}\right.$} & 2 & 91 \\
\hline
\end{tabular}

a Time for total completion of the reaction.

${ }^{\mathrm{b}}$ Isolated yield.

TABLE 2: Recycling of $\left[\mathrm{Bmim}^{-B_{4}} \mathrm{~B}_{4}\right.$ ionic liquid.

\begin{tabular}{lccc}
\hline Entry & Cycle & Time $(\mathrm{h})$ & ${\text { Yield }(\%)^{\mathrm{a}}}^{\mathrm{a}}$ \\
\hline 1 & 1st run & 2 & 91 \\
2 & 2nd run & 2 & 90 \\
3 & 3rd run & 2 & 88 \\
4 & 4th run & 2.5 & 87 \\
\hline
\end{tabular}

Isolated yield of $\mathbf{6 b}$.

of novel sulfanyl tetrazoles using $[\mathrm{Bmim}] \mathrm{BF}_{4}$ ionic liquid. This protocol has the advantages of simple workup, short reaction times, milder reaction conditions, good yields, and environmentally benign reusable solvent.

\section{Experimental}

4.1. General. Melting points were recorded in open capillary and were uncorrected. Column chromatography was performed using silicagel (100-200 mesh size) purchased from Thomas Baker and TLC was carried out using aluminium sheets precoated with silica gel 60F254 purchased from Merck. IR spectra (KBr) were recorded on a Bruker WM$4(\mathrm{X})$ spectrometer (577 model). ${ }^{1} \mathrm{H}$ NMR $(300 \mathrm{MHz})$ and ${ }^{13} \mathrm{C}$ NMR $(75 \mathrm{MHz})$ spectra were recorded on Bruker AC300 spectrometer in $\mathrm{CDCl}_{3}$ and $\mathrm{DMSO}-d_{6}$ with TMS as an internal standard. Mass spectra (ESI) were recorded on JEOL SX-102 spectrometer. CHN analysis was done by Carlo Erba EA 1108 automatic elemental analyzer. The chemicals and solvents used were of commercial grade and were used without further purification unless, otherwise, stated.

4.2. Typical Procedure. A mixture of phenacylbromide $5 \mathbf{a}-\mathbf{f}$ (1 mmol) or 3-(2-bromoacetyl)coumarin 1a-f $(1 \mathrm{mmol})$ and $\mathrm{KSCN}(1.2 \mathrm{mmol})$ in $[\mathrm{Bmim}] \mathrm{BF}_{4}(3 \mathrm{~mL})$ was stirred at RT for $10 \mathrm{~min}$ (in case of 3-(2-bromoacetyl)coumarins; $45 \mathrm{~min}$ ), after completion of the reaction (single spot on TLC), $\mathrm{NaN}_{3}$ $(1.2 \mathrm{mmol})$ was added portion wise to the reaction mixture and then it was stirred at $100^{\circ} \mathrm{C}$ for appropriate time. After completion of the reaction (monitored by TLC), it was cooled to RT and poured into ice cold water, the solid separated was filtered, washed with water, dried, and purified by column chromatography using silicagel (ethylacetate/n-hexane: $2 / 8$ ) to afford title compounds $\mathbf{4 a - f}$ and $\mathbf{6 a}-\mathbf{f}$ in good yields.

3-(2-(1H-Tetrazol-5-ylsulfanyl)-acetyl)-chromen-2-one (4a). m.p. $154-156^{\circ} \mathrm{C}$; IR $\left(\mathrm{KBr}, \mathrm{cm}^{-1}\right)$ 3347, 3073, 1729, 1680, 
<smiles></smiles>

SCHEME 1<smiles>[R]c1ccc(C(=O)CSc2nnn[nH]2)cc1</smiles>

SCHEMe 2

1551; ${ }^{1} \mathrm{H}$ NMR $\left(300 \mathrm{MHz}, \mathrm{CDCl}_{3}\right) \delta 4.96\left(\mathrm{~s}, 2 \mathrm{H},-\mathrm{CH}_{2}\right)$, 7.34-7.36 (m, 2H, ArH), 7.95 (d, 2H, ArH), 8.80 (s, 1H, C of coumarin); ${ }^{13} \mathrm{C} \mathrm{NMR}\left(75 \mathrm{MHz}, \mathrm{CDCl}_{3}\right): \delta 42.89,123.21$, $125.22,128.32,129.65,130.36,135.15,145.16,148.84$, 155.18, 158.94, 185.66; MS m/z: $289(\mathrm{M}+1)^{+}$; Anal. Calcd for $\mathrm{C}_{12} \mathrm{H}_{8} \mathrm{~N}_{4} \mathrm{O}_{3} \mathrm{~S}$; C, 50.00; H, 2.80; N, 19.43. Found: C, 50.12; $\mathrm{H}, 2.71 ; \mathrm{N}, 19.37 \%$.

6-Chloro-3-(2-(1H-tetrazol-5-ylsulfanyl)-acetyl)-chromen-2one (4b). m.p. $161-163^{\circ} \mathrm{C}$; IR $\left(\mathrm{KBr}, \mathrm{cm}^{-1}\right) 3342,3075,1719$, 1676, 1554; ${ }^{1} \mathrm{H}$ NMR $\left(300 \mathrm{MHz}, \mathrm{CDCl}_{3}\right) \delta 4.94$ (s, 2H, $\left.-\mathrm{CH}_{2}\right), 7.44-7.46(\mathrm{~m}, 2 \mathrm{H}, \mathrm{ArH}), 7.96$ (s, 1H, ArH), 8.82 (s, $1 \mathrm{H}, \mathrm{C}_{4}$ of coumarin); ${ }^{13} \mathrm{C} \mathrm{NMR}\left(75 \mathrm{MHz}, \mathrm{CDCl}_{3}\right) \delta$ 42.64, 125.34, 128.41, 129.65, 130.36, 131.52, 135.11, 145.05, 147.89, 154.52, 158.58, 184.37; MS m/z: $323(\mathrm{M}+1)^{+}$; Anal. Calcd for $\mathrm{C}_{12} \mathrm{H}_{7} \mathrm{ClN}_{4} \mathrm{O}_{3} \mathrm{~S}: \mathrm{C}, 44.66 ; \mathrm{H}, 2.19 ; \mathrm{N}, 17.36$. Found: C, $44.52 ; \mathrm{H}, 2.26 ; \mathrm{N}, 17.40 \%$.

6,8-Dichloro-3-(2-(1H-tetrazol-5-ylsulfanyl)-acetyl)-chromen2-one (4c). m.p. $173-175^{\circ} \mathrm{C}$; IR $\left(\mathrm{KBr}, \mathrm{cm}^{-1}\right) 3347,3071$, 1711, 1672, 1557; ${ }^{1} \mathrm{H}$ NMR (300 MHz, $\left.\mathrm{CDCl}_{3}\right) \delta 4.94(\mathrm{~s}$, $2 \mathrm{H},-\mathrm{CH}_{2}$ ), 7.58 (s, 1H, ArH), 7.84 (s, 1H, ArH), 8.80 (s, $1 \mathrm{H}, \mathrm{C}_{4}$ of coumarin); ${ }^{13} \mathrm{C} \mathrm{NMR}\left(75 \mathrm{MHz}, \mathrm{CDCl}_{3}\right) \delta 42.66$, $124.16,127.93,129.42,131.35,134.32,136.17,142.86,146.91$, 154.56, 158.77 184.37; MS m/z: $358(\mathrm{M}+1)^{+}$; Anal. Calcd for $\mathrm{C}_{12} \mathrm{H}_{6} \mathrm{Cl}_{2} \mathrm{~N}_{4} \mathrm{O}_{3} \mathrm{~S}$ : C, 40.35; H, 1.69; $\mathrm{N}, 19.85$. Found: $\mathrm{C}$, 40.46; H, 1.61 ; N, 19.80\%.

6-Bromo-3-(2-(1H-tetrazol-5-ylsulfanyl)-acetyl)-chromen-2one (4d). m.p. $146-148^{\circ} \mathrm{C}$; IR $\left(\mathrm{KBr}, \mathrm{cm}^{-1}\right) 3344,3073,1729$, 1680, 1562; ${ }^{1} \mathrm{H}$ NMR $\left(300 \mathrm{MHz}, \mathrm{CDCl}_{3}\right) \delta 4.96(\mathrm{~s}, 2 \mathrm{H}$, $\left.-\mathrm{CH}_{2}\right), 7.44-7.46(\mathrm{~m}, 2 \mathrm{H}, \mathrm{ArH}), 7.95$ (s, 1H, ArH), 8.84 (s, $1 \mathrm{H}, \mathrm{C}_{4}$ of coumarin); ${ }^{13} \mathrm{C} \mathrm{NMR}\left(75 \mathrm{MHz}, \mathrm{CDCl}_{3}\right) \delta$ $42.78,120.19,127.91,129.89,130.78,132.44,136.31,146.75$, 149.03, 154.62, 158.71 184.43; MS m/z: $368(\mathrm{M}+1)^{+}$; Anal. Calcd for $\mathrm{C}_{12} \mathrm{H}_{7} \mathrm{BrN}_{4} \mathrm{O}_{3} \mathrm{~S}$ : C, 39.25; $\mathrm{H}, 1.92 ; \mathrm{N}, 15.26$. Found C, 39.18; H, 1.98; N, 15.20\%.

6,8-Dibromo-3-(2-(1H-tetrazol-5-ylsulfanyl)-acetyl)-chromen-2-one (4e). m.p. $167-169^{\circ} \mathrm{C}$; IR ( $\left.\mathrm{KBr}, \mathrm{cm}^{-1}\right) 3341,3078$,
1714, 1677, 1557; ${ }^{1} \mathrm{H}$ NMR (300 MHz, $\left.\mathrm{CDCl}_{3}\right) \delta 4.92$ (s, $2 \mathrm{H},-\mathrm{CH}_{2}$ ), 7.62 (s, 1H, ArH), 7.85 (s, 1H, ArH), 8.80 (s, $1 \mathrm{H}, \mathrm{C}_{4}$ of coumarin); ${ }^{13} \mathrm{C} \mathrm{NMR}\left(75 \mathrm{MHz}, \mathrm{CDCl}_{3}\right) \delta 42.70$, 123.26, 128.71, 129.83, 131.47, 133.21, 136.31, 143.47, 147.82, 154.48, 158.63 184.32; MS m/z: $447(\mathrm{M}+1)^{+}$; Anal. Calcd for $\mathrm{C}_{12} \mathrm{H}_{6} \mathrm{Br}_{2} \mathrm{~N}_{4} \mathrm{O}_{3} \mathrm{~S}$ : C, 32.31; $\mathrm{H}, 1.36 ; \mathrm{N}, 12.56$. Found: $\mathrm{C}$, $32.44 ; \mathrm{H}, 1.28 ; \mathrm{N}, 12.50 \%$.

8-Methoxy-3-(2-(1H-tetrazol-5-ylsulfanyl)-acetyl)-chromen2-one (4f). m.p. $131-133^{\circ} \mathrm{C}$; IR $\left(\mathrm{KBr}, \mathrm{cm}^{-1}\right) v=3346,3080$, 1724, 1684, 1566; ${ }^{1} \mathrm{H}$ NMR $\left(300 \mathrm{MHz}, \mathrm{CDCl}_{3}\right) \delta 3.78(\mathrm{~s}, 3 \mathrm{H}$, $-\mathrm{OCH}_{3}$ ), $4.90\left(\mathrm{~s}, 2 \mathrm{H},-\mathrm{CH}_{2}\right), 7.22-7.30(\mathrm{~m}, 3 \mathrm{H}, \mathrm{ArH}), 8.82$ (s, $1 \mathrm{H}, \mathrm{C}_{4}$ of coumarin); ${ }^{13} \mathrm{C} \mathrm{NMR}\left(75 \mathrm{MHz}, \mathrm{CDCl}_{3}\right) \delta 42.94$, 55.71, 115.93, 121.17, 123.21, 129.65, 130.24, 134.11, 137.16, 154.38, 157.84, 160.21, 185.66; MS m/z: $319(\mathrm{M}+1)^{+}$; Anal. Calcd. for $\mathrm{C}_{13} \mathrm{H}_{10} \mathrm{~N}_{4} \mathrm{O}_{4} \mathrm{~S}$ : C, 49.05; H, 3.17; N, 17.60. Found: C, $49.12 ; \mathrm{H}, 3.24 ; \mathrm{N}, 17.52 \%$.

1-Phenyl-2-(1H-tetrazol-5-ylsulfanyl)-ethanone (6a). m.p. 143-145 $\mathrm{C}$; IR $\left(\mathrm{KBr}, \mathrm{cm}^{-1}\right) 3423,3076,1672,1588 ;{ }^{1} \mathrm{H}$ NMR $\left(300 \mathrm{MHz}, \mathrm{DMSO}-d_{6}\right) \delta 4.90\left(\mathrm{~s}, 2 \mathrm{H},-\mathrm{CH}_{2}\right), 7.66-7.95(\mathrm{~m}$, $5 \mathrm{H}, \mathrm{ArH}) ;{ }^{13} \mathrm{C}$ NMR $\left(75 \mathrm{MHz}, \mathrm{DMSO}-d_{6}\right): \delta 49.23,128.82$, 131.43, 133.97, 139.82, 151.73, 192.12; MS $m / z=221[\mathrm{M}+1]^{+}$; Anal. Calcd. for $\mathrm{C}_{9} \mathrm{H}_{8} \mathrm{~N}_{4}$ OS: C, 49.08; H, 3.66; N, 25.44. Found: C, $49.21 ; \mathrm{H}, 3.51 ; \mathrm{N}, 25.39 \%$.

1-(4-Chlorophenyl)-2-(1H-tetrazol-5-ylsulfanyl)-ethanone (6b). m.p. $166-168^{\circ} \mathrm{C}$; IR $\left(\mathrm{KBr}, \mathrm{cm}^{-1}\right) v=3417,3070$, 1678, 1593; ${ }^{1} \mathrm{H}$ NMR $\left(300 \mathrm{MHz}, \mathrm{DMSO}-d_{6}\right): \delta 5.04(\mathrm{~s}$, $\left.2 \mathrm{H},-\mathrm{CH}_{2}\right), 7.71$ (d, 2H, ArH), 8.10 (d, $\left.2 \mathrm{H}, \mathrm{ArH}\right) ;{ }^{13} \mathrm{C}$ NMR $\left(75 \mathrm{MHz}, \mathrm{DMSO}-d_{6}\right): \delta 48.05,128.95,130.32,133.84$, 138.76, 151.58, 191.94; MS $m / z=255[\mathrm{M}+1]^{+}$; Anal. Calcd. for $\mathrm{C}_{9} \mathrm{H}_{7} \mathrm{ClN}_{4} \mathrm{OS}$ : C, 42.44; $\mathrm{H}, 2.77 ; \mathrm{N}, 22.00$. Found: C, 42.53; H, 2.89; N, 21.87\%.

1-(4-Bromophenyl)-2-(1H-tetrazol-5-ylsulfanyl)-ethanone (6c). m.p. $178-180^{\circ} \mathrm{C}$; IR $\left(\mathrm{KBr}, \mathrm{cm}^{-1}\right) 3420,3079,1678$, 1598; ${ }^{1} \mathrm{H}$ NMR $\left(300 \mathrm{MHz}, \mathrm{DMSO}-d_{6}\right) \delta 5.08\left(\mathrm{~s}, 2 \mathrm{H},-\mathrm{CH}_{2}\right)$, 7.75 (d, 2H, ArH), 8.12 (d, 2H, ArH); ${ }^{13} \mathrm{C}$ NMR $(75 \mathrm{MHz}$, DMSO- $\left.d_{6}\right) \delta 48.04,126.92,130.48,131.54,136.32,151.68$, 
TABLE 3: Synthesis of sulfanyl tetrazoles $\mathbf{4 a}-\mathbf{f}$ and $\mathbf{6 a}-\mathbf{f}$ in $[\mathrm{Bmim}] \mathrm{BF}_{4}$ ionic liquid.

\begin{tabular}{|c|c|c|c|c|c|c|}
\hline Entry & $\mathrm{R}$ & $\mathrm{R}_{1}$ & $\mathrm{R}_{2}$ & Product & Time (h) ${ }^{\mathrm{a}}$ & Yield (\%) \\
\hline 1 & - & $\mathrm{H}$ & $\mathrm{H}$ & $4 a$ & 4 & 88 \\
\hline 2 & - & $\mathrm{H}$ & $\mathrm{Cl}$ & $4 b$ & 4.5 & 85 \\
\hline 3 & - & $\mathrm{Cl}$ & $\mathrm{Cl}$ & $4 c$ & 4.5 & 78 \\
\hline 4 & - & $\mathrm{H}$ & $\mathrm{Br}$ & $4 d$ & 5 & 82 \\
\hline 5 & - & $\mathrm{Br}$ & $\mathrm{Br}$ & $4 e$ & 5.5 & 75 \\
\hline 6 & - & $\mathrm{OMe}$ & $\mathrm{H}$ & $4 \mathrm{f}$ & 6 & 81 \\
\hline 7 & $\mathrm{H}$ & - & - & $6 a$ & 3 & 90 \\
\hline 8 & 4-Cl & - & - & $6 b$ & 2 & 91 \\
\hline 9 & $4-\mathrm{Br}$ & - & - & $6 c$ & 3 & 88 \\
\hline 10 & $4-\mathrm{Me}$ & - & - & $6 d$ & 3.5 & 83 \\
\hline 11 & 4 -OMe & - & - & $6 e$ & 4 & 86 \\
\hline 12 & $4-\mathrm{NO}_{2}$ & - & - & $6 f$ & 2.5 & 80 \\
\hline
\end{tabular}

a Time for total completion of the reaction.

${ }^{\mathrm{b}}$ Isolated yield.

191.93; MS m/z: $300(\mathrm{M}+1)^{+}$; Anal. Calcd for $\mathrm{C}_{9} \mathrm{H}_{7} \mathrm{BrN}_{4} \mathrm{OS}$ : C, 36.13; H, 2.36; N, 18.73. Found: C, 36.22; H, 2.27; N, $18.63 \%$.

1-(4-Methylphenyl)-2-(1H-tetrazol-5-ylsulfanyl)-ethanone (6d). m.p. $161-163^{\circ} \mathrm{C}$; IR $\left(\mathrm{KBr}, \mathrm{cm}^{-1}\right) 3388,3073,1670$, $1571 ;{ }^{1} \mathrm{H}$ NMR $\left(300 \mathrm{MHz}, \mathrm{DMSO}-d_{6}\right) \delta 2.42\left(\mathrm{~s}, 3 \mathrm{H}, \mathrm{CH}_{3}\right)$ $4.84\left(\mathrm{~s}, 2 \mathrm{H},-\mathrm{CH}_{2}\right), 7.62-7.84(\mathrm{~m}, 4 \mathrm{H}, \mathrm{ArH}) ;{ }^{13} \mathrm{C} \mathrm{NMR}$ $\left(75 \mathrm{MHz}, \mathrm{DMSO}-d_{6}\right) \delta 25.30,47.82,126.94,130.27,134.78$, 142.84, 151.18, 191.87; MS m/z: $235(\mathrm{M}+1)^{+}$; Anal. Calcd for $\mathrm{C}_{10} \mathrm{H}_{10} \mathrm{~N}_{4} \mathrm{OS}$ : C, 51.27; H, 4.30; N, 23.91. Found: C, 51.36; $\mathrm{H}, 4.22 ; \mathrm{N}, 23.98 \%$.

1-(4-Methoxyphenyl)-2-(1H-tetrazol-5-ylsulfanyl)-ethanone (6e). m.p. $150-152^{\circ} \mathrm{C}$; IR $\left(\mathrm{KBr}, \mathrm{cm}^{-1}\right) 3443,3091,1671$, $1578 ;{ }^{1} \mathrm{H}$ NMR $\left(300 \mathrm{MHz}, \mathrm{DMSO}-d_{6}\right) \delta 3.75\left(\mathrm{~s}, 3 \mathrm{H}, \mathrm{OCH}_{3}\right)$, $4.96\left(\mathrm{~s}, 2 \mathrm{H},-\mathrm{CH}_{2}\right), 7.67(\mathrm{~d}, 2 \mathrm{H}, \mathrm{ArH}), 7.82(\mathrm{~d}, 2 \mathrm{H}, \mathrm{ArH}) ;{ }^{13} \mathrm{C}$ NMR $\left(75 \mathrm{MHz}\right.$, DMSO- $\left.d_{6}\right) \delta 48.16,55.91,120.26,129.47$, 131.52, 151.02, 162.73, 191.70; MS $\mathrm{m} / z: 251(\mathrm{M}+1)^{+}$; Anal. Calcd for $\mathrm{C}_{10} \mathrm{H}_{10} \mathrm{~N}_{4} \mathrm{O}_{2} \mathrm{~S}$ : C, 47.99; $\mathrm{H}, 4.03 ; \mathrm{N}, 22.39$. Found: C, $47.86 ; \mathrm{H}, 3.96 ; \mathrm{N}, 22.32 \%$.

1-(4-Nitrophenyl)-2-(1H-tetrazol-5-ylsulfanyl)-ethanone (6f). m.p. $176-178^{\circ} \mathrm{C}$; IR $\left(\mathrm{KBr}, \mathrm{cm}^{-1}\right) 3341,3094,1680,1574 ;{ }^{1} \mathrm{H}$ NMR $\left(300 \mathrm{MHz}\right.$, DMSO- $\left.d_{6}\right) \delta 5.10\left(\mathrm{~s}, 2 \mathrm{H},-\mathrm{CH}_{2}\right), 7.80-8.02$ $(\mathrm{m}, 4 \mathrm{H}, \mathrm{ArH}) ;{ }^{13} \mathrm{C}$ NMR $\left(75 \mathrm{MHz}, \mathrm{DMSO}-d_{6}\right) \delta 48.07$, 127.14, 129.67, 141.36, 150.12, 152.03, 191.78; MS m/z: 266 $(\mathrm{M}+1)^{+}$; Anal. Calcd for $\mathrm{C}_{9} \mathrm{H}_{7} \mathrm{~N}_{5} \mathrm{O}_{3} \mathrm{~S}: \mathrm{C}, 40.75 ; \mathrm{H}, 2.66 ; \mathrm{N}$, 26.40. Found: C, 40.86; H, 2.62; N, 26.31\%.

\section{Acknowledgments}

The authors are thankful to the Director, National Institute of Technology-Warangal, for providing research facilities and financial support.

\section{References}

[1] J. Zhu and H. Bienayme, Multicomponent Reactions, WileyVCH, Weinheim, Germany, 2005.

[2] A. Dömling, "Recent developments in isocyanide based multicomponent reactions in applied chemistry," Chemical Reviews, vol. 106, no. 1, pp. 17-89, 2006.

[3] R. N. Butler, A. R Katritzky, C. W Rees, and E. F. V Scriven, Comprehensive Heterocyclic Chemistry, Pergamon, Oxford, UK, 1996.

[4] R. Jason Herr, "5-Substituted-1H-tetrazoles as carboxylic acid isosteres: medicinal chemistry and synthetic methods," Bioorganic and Medicinal Chemistry, vol. 10, no. 11, pp. 3379-3393, 2002.

[5] H. Singh, A. Singh Chawla, V. K. Kapoor, D. Paul, and R. K. Malhotra, "4 Medicinal chemistry of tetrazoles," Progress in Medicinal Chemistry, vol. 17, pp. 151-183, 1980.

[6] G. F. Holland and J. N. Pereira, "Heterocyclic tetrazoles, a new class of lipolysis inhibitors," Journal of Medicinal Chemistry, vol. 10, no. 2, pp. 149-154, 1967.

[7] D. W. Esplin and D. M. Woodbury, "The fate and excretion of C14-labeled pentylenetetrazol in the rat, with comments on analytical methods for pentylenetetrazol," The Journal of Pharmacology and Experimental Therapeutics, vol. 118, no. 2, pp. 129-138, 1956.

[8] H. Eshghi and A. Hassankhani, "Preparation of 1,5-fused tetrazoles under solvent-free conditions," Synthetic Communications, vol. 35, pp. 1115-1120, 2005.

[9] L. A. Flippin, "Directed metalation and new synthetic transformations of 5-aryltetrazoles," Tetrahedron Letters, vol. 32, no. 47, pp. 6857-6860, 1991.

[10] P. Rhonnstad and D. Wensbo, "On the relative strength of the $1 \mathrm{H}$-tetrazol-5-yl- and the 2-(triphenylmethyl)-2H-tetrazol-5yl-group in directed ortho-lithiation," Tetrahedron Letters, vol. 43, no. 17, pp. 3137-3139, 2002.

[11] F. Ek, L.-G. Wistrand, and T. Frejd, "Synthesis of fused tetrazoleand imidazole derivatives via iodocyclization," Tetrahedron, vol. 59, no. 35, pp. 6759-6769, 2003. 
[12] G. Sandmann, C. Schneider, and P. Boger, "A new nonradioactive assay of phytoene desaturase to evaluate bleaching herbicides," vol. 51, no. 7-8, pp. 534-538, 1996.

[13] B. S. Jursic and B. W. Le Blanc, "Preparation of tetrazoles from organic nitriles and sodium azide in micellar media," Journal of Heterocyclic Chemistry, vol. 35, no. 2, pp. 405-408, 1998.

[14] F. Wincott, A. DiRenzo, C. Shaffer et al., "Synthesis, deprotection, analysis and purification of RNA and ribozymes," Nucleic Acids Research, vol. 23, no. 14, pp. 2677-2684, 1995.

[15] D. Tsou, A. Hampel, A. Andrus, and R. Vinayak, "Large scale synthesis of oligoribonucleotides on high-loaded polystyrene (HLP) support," Nucleosides and Nucleotides, vol. 14, no. 7, pp. 1481-1492, 1995.

[16] E. Lieber and T. Enkoji, "Synthesis and properties of 5(substituted) mercaptotetrazoles," Journal of Organic Chemistry, vol. 26, no. 11, pp. 4472-4479, 1961.

[17] M. Curini, G. Cravotto, F. Epifano, and G. Giannone, "Chemistry and biological activity of natural and synthetic prenyloxycoumarins," Current Medicinal Chemistry, vol. 13, no. 2, pp. 199-222, 2006.

[18] F. Borges, F. Roleira, N. Milhazes, L. Santana, and E. Uriarte, "Simple coumarins and analogues in medicinal chemistry: occurrence, synthesis and biological activity," Current Medicinal Chemistry, vol. 12, no. 8, pp. 887-916, 2005.

[19] K. C. Fylaktakidou, D. J. Hadjipavlou-Litina, K. E. Litinas, and D. N. Nicolaides, "Natural and synthetic coumarin derivatives with anti-inflammatory/antioxidant activities," Current Pharmaceutical Design, vol. 10, no. 30, pp. 3813-3833, 2004.

[20] L. Xie, Y. Takeuchi, L. M. Cosentino, A. T. McPhail, and K.H. Lee, "Anti-AIDS agents. 42. Synthesis and anti-HIV activity of disubstituted ( $\left.3^{\prime} \mathrm{R}, 4^{\prime} \mathrm{R}\right)-3^{\prime}, 4^{\prime}$-di-O-(S)-camphanoyl-(+)-ciskhellactone analogues," Journal of Medicinal Chemistry, vol. 44, no. 5, pp. 664-671, 2001.

[21] J. V. Duncia, M. E. Pierce, and J. B. Santella III, "Three synthetic routes to a sterically hindered tetrazole. A new one-step mild conversion of an amide into a tetrazole," Journal of Organic Chemistry, vol. 56, no. 7, pp. 2395-2400, 1991.

[22] K. Sisido, K. Nabika, T. Isida, and S. Kozima, "Formation of organo tin-nitrogen bonds. III. N-trial-kyltin-5-substituted tetrazoles," Journal of Organometallic Chemistry, vol. 33, no. 3, pp. 337-346, 1971.

[23] D. J. Carini, J. V. Duncia, P. E. Aldrich et al., "Nonpeptide angiotensin II receptor antagonists: the discovery of a series of $\mathrm{N}$-(biphenylylmethyl)imidazoles as potent, orally active antihypertensives," Journal of Medicinal Chemistry, vol. 34, no. 8, pp. 2525-2547, 1991.

[24] S. J. Wittenberger and B. G. Donner, "Dialkyltin oxide mediated addition of trimethylsilyl azide to nitriles. A novel preparation of 5-substituted tetrazoles," Journal of Organic Chemistry, vol. 58, no. 15, pp. 4139-4141, 1993.

[25] S. Rostamizadeh, H. Ghaieni, R. Aryan, and A. Amani, "Zinc chloride catalyzed synthesis of 5 -substituted $1 \mathrm{H}$-tetrazoles under solvent free condition," Chinese Chemical Letters, vol. 20, no. 11, pp. 1311-1314, 2009.

[26] D. P. Matthews, J. E. Green, and A. J. Shuker, "Parallel synthesis of alkyl tetrazole derivatives using solid support chemistry," Journal of Combinatorial Chemistry, vol. 2, no. 1, pp. 19-23, 2000.

[27] A. Kumar, R. Narayanan, and H. Shechter, "Rearrangement reactions of (hydroxyphenyl)carbenes," Journal of Organic Chemistry, vol. 61, no. 13, pp. 4462-4465, 1996.
[28] M. Nasrollahzadeh, Y. Bayat, D. Habibi, and S. Moshaee, " $\mathrm{FeCl}_{3}-\mathrm{SiO}_{2}$ as a reusable heterogeneous catalyst for the synthesis of 5-substituted $1 \mathrm{H}$-tetrazoles via [2+3] cycloaddition of nitriles and sodium azide," Tetrahedron Letters, vol. 50, no. 31, pp. 4435-4438, 2009.

[29] Y. S. Gyoung, J. G. Shim, and Y. Yamamoto, "Regiospecific synthesis of 2-allylated-5-substituted tetrazoles via palladiumcatalyzed reaction of nitriles, trimethylsilyl azide, and allyl acetates," Tetrahedron Letters, vol. 41, no. 21, pp. 4193-4196, 2000.

[30] D. Amantini, R. Beleggia, F. Fringuelli, F. Pizzo, and L. Vaccaro, "TBAF-catalyzed synthesis of 5-substituted $1 \mathrm{H}$-tetrazoles under solventless conditions," Journal of Organic Chemistry, vol. 69, no. 8, pp. 2896-2898, 2004.

[31] M. L. Kantam, K. B. Shiva Kumar, and K. Phani Raja, "An efficient synthesis of 5-substituted $1 \mathrm{H}$-tetrazoles using $\mathrm{Zn} / \mathrm{Al}$ hydrotalcite catalyst," Journal of Molecular Catalysis A, vol. 247, no. 1-2, pp. 186-188, 2006.

[32] M. Lakshmi Kantam, K. B. Shiva Kumar, and Ch. Sridhar, "Nanocrystalline $\mathrm{ZnO}$ as an efficient heterogeneous catalyst for the synthesis of 5-substituted $1 \mathrm{H}$-tetrazoles," Advanced Synthesis and Catalysis, vol. 347, no. 9, pp. 1212-1214, 2005.

[33] T. Jin, F. Kitahara, S. Kamijo, and Y. Yamamoto, "Coppercatalyzed synthesis of 5-substituted $1 \mathrm{H}$-tetrazoles via the [3+2] cycloaddition of nitriles and trimethylsilyl azide," Tetrahedron Letters, vol. 49, no. 17, pp. 2824-2827, 2008.

[34] B. W. LeBlanc and B. S. Jursic, "Preparation of 5-alkylthio and 5arylthiotetrazoles from thiocyanates using phase transfer catalysis," Synthetic Communications, vol. 28, no. 19, pp. 3591-3599, 1998.

[35] Z. P. Demko and K. B. Sharpless, "Preparation of 5-substituted $1 \mathrm{H}$-tetrazoles from nitriles in water," Journal of Organic Chemistry, vol. 66, no. 24, pp. 7945-7950, 2001.

[36] P. Wasserscheid and T. Welton, Ionic Liquids in Synthesis, WileyVCH, Weinheim, Germany, 2003.

[37] R. Sheldon, "Catalytic reactions in ionic liquids," Chemical Communications, no. 23, pp. 2399-2407, 2001.

[38] T. Welton, "Room-temperature ionic liquids. Solvents for synthesis and catalysis," Chemical Reviews, vol. 99, no. 8, pp. 2071-2083, 1999.

[39] J. S. Wilkes, "A short history of ionic liquids-from molten salts to neoteric solvents," Green Chemistry, vol. 4, no. 2, pp. 73-80, 2002.

[40] N. Jain, A. Kumar, S. Chauhan, and S. M. S. Chauhan, "Chemical and biochemical transformations in ionic liquids," Tetrahedron, vol. 61, no. 5, pp. 1015-1060, 2005.

[41] A. R. Gholap, K. Venkatesan, T. Daniel, R. J. Lahoti, and K. V. Srinivasan, "Ionic liquid promoted novel and efficient one pot synthesis of 3,4-dihydropyrimidin-2-(1H)-ones at ambient temperature under ultrasound irradiation," Green Chemistry, vol. 6, no. 3, pp. 147-150, 2004.

[42] R. N. Nadaf, S. A. Siddiqui, T. Daniel, R. J. Lahoti, and K. V. Srinivasan, "Room temperature ionic liquid promoted regioselective synthesis of 2-aryl benzimidazoles, benzoxazoles and benzthiazoles under ambient conditions," Journal of Molecular Catalysis A, vol. 214, no. 1, pp. 155-160, 2004.

[43] S. A. Siddiqui, T. M. Potewar, R. J. Lahoti, and K. V. Srinivasan, "Ionic liquid promoted facile one-pot synthesis of 1pyridylimidazo[1,5-a] pyridines from dipyridylketone and aryl aldehydes," Synthesis, no. 17, pp. 2849-2854, 2006. 
[44] S. A. Siddiqui, U. C. Narkhede, S. S. Palimkar, T. Daniel, R. J. Lahoti, and K. V. Srinivasan, "Room temperature ionic liquid promoted improved and rapid synthesis of 2,4,5-triaryl imidazoles from aryl aldehydes and 1,2-diketones or $\alpha$ hydroxyketone," Tetrahedron, vol. 61, no. 14, pp. 3539-3546, 2005.

[45] M. B. Maradolla, S. K. Allam, A. Mandha, and G. V. P. Chandramouli, "One pot synthesis of benzoxazoles, benzthiazoles and benzimidazoles from carboxylic acids using ionic liquids," Arkivoc, vol. 2008, no. 15, pp. 42-46, 2008.

[46] S. Kanakaraju, B. Prasanna, S. Basavoju, and G. V. P. Chandramouli, "Ionic liquid catalyzed one-pot multi-component synthesis, characterization and antibacterial activity of novel chromeno[2,3-d]pyrimidin-8-amine derivatives," Journal of Molecular Structure, vol. 1017, pp. 60-64, 2012. 

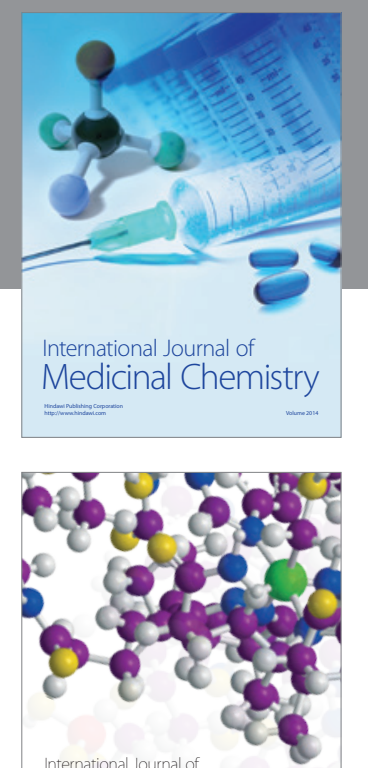

\section{Carbohydrate} Chemistry

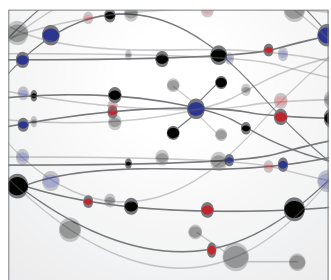

The Scientific World Journal
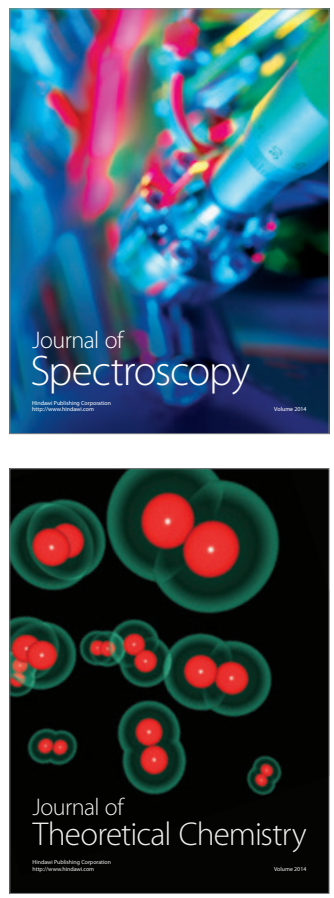
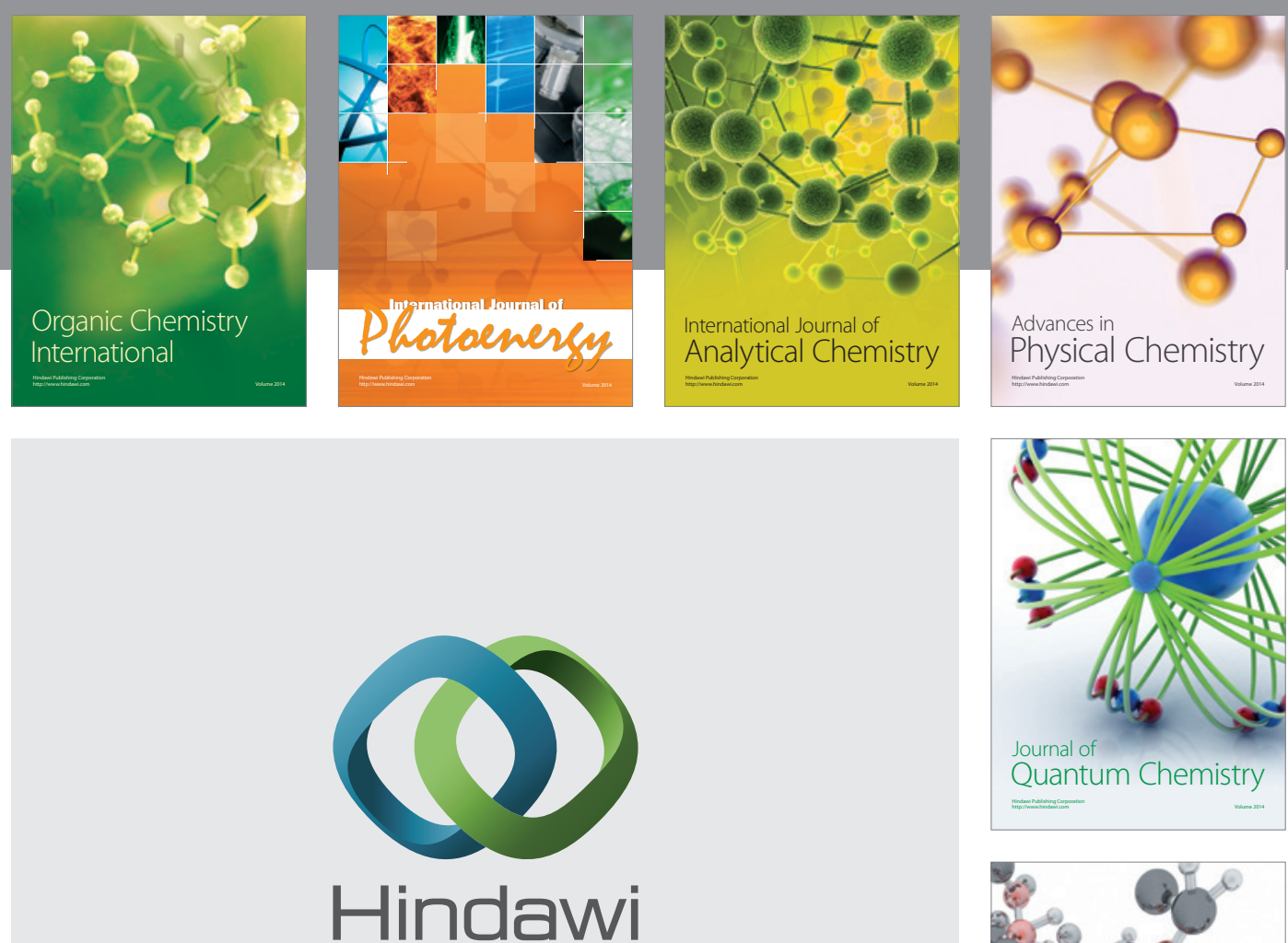

Submit your manuscripts at

http://www.hindawi.com

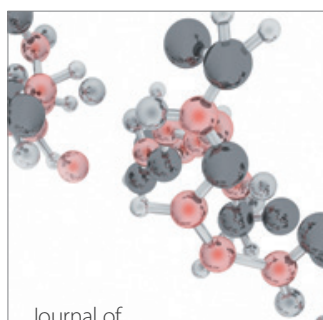

Analytical Methods

in Chemistry

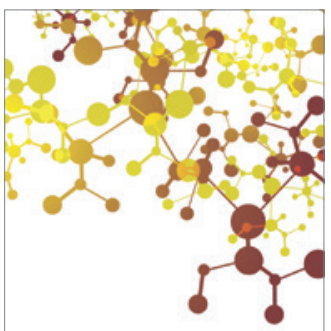

Journal of

Applied Chemistry

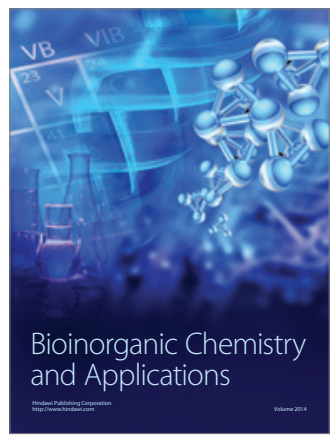

Inorganic Chemistry
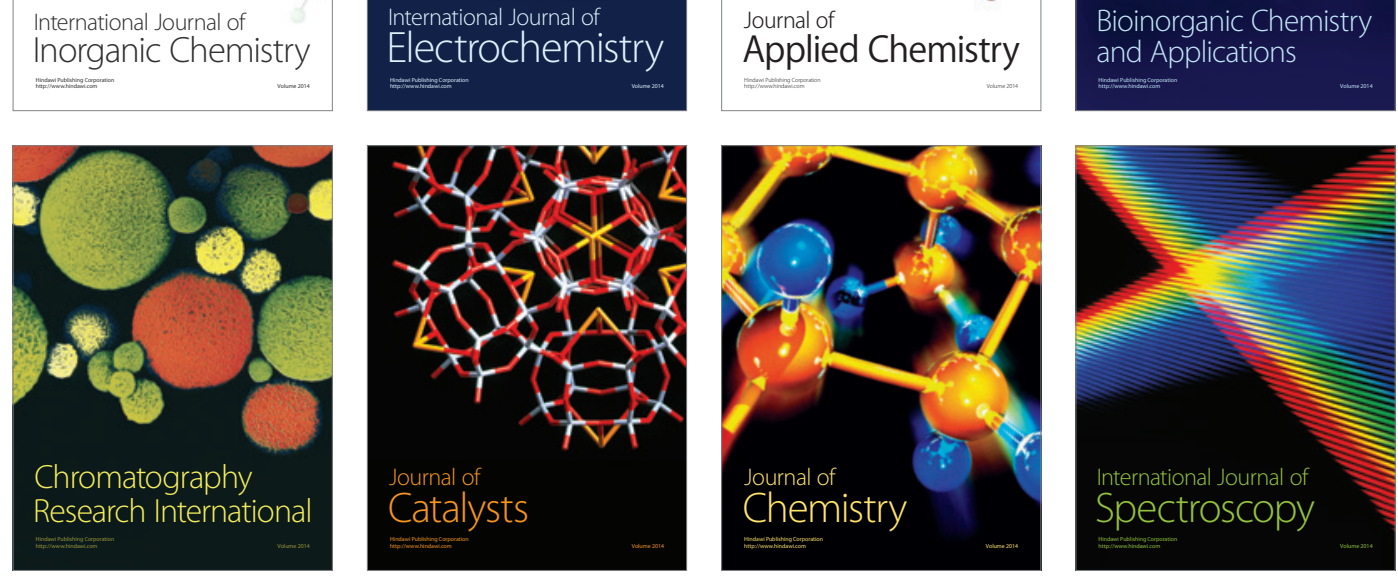